\title{
10GHZ SKY SURVEY PROJECT, WASEDA FFT INTERFEROMETER
}

\author{
J. NAKAJIMA ${ }^{1}$, E. OTOBE, K. ASUMA ${ }^{2}$ \\ Astrophysics, Department of Pure and Applied physics, \\ Waseda University, Okubo Shinjuku-ku Tokyo 169, Japan
}

\section{T. DAISHIDO}

Astrophysics, Department of Science, School of Education, Waseda University, Nishi-Waseda Shinjuku-ku Tokyo 169, Japan

\section{INTRODUCTION}

Large-field radio interferometer at $10.65 \mathrm{GHz}$ have been developed to search for transient radio objects such as radio supernovae and radio bursts in stellar systems (Daishido et al. 1984). This is a spatial fast-fourier transform (FFT) type radio interferometer, being an equally-spaced, maximum redundant, twodimensional (2D) array in an $8 \times 8$ configuration. Sixty-four identically-designed frontend elements are comprised of $2.4 \mathrm{~m}$ diameter cassegrain antennas and $200 \mathrm{~K}$ HEMT receivers. These are steerable in elevetion and are fixed in azimuth. Although it is only partially operating, the completed system having 64 beams in the northern hemisphere is expected to provide maps having $0.1^{\circ}$ angular resolution and a sensitivity of $50 \mathrm{mJy}$. The beams are formed by a newly-developed "Digital Lens" (complex amplitude equalizer + 2D FFT pipelined processor), with the array's overall size being $20 \times 20 \mathrm{~m}$.

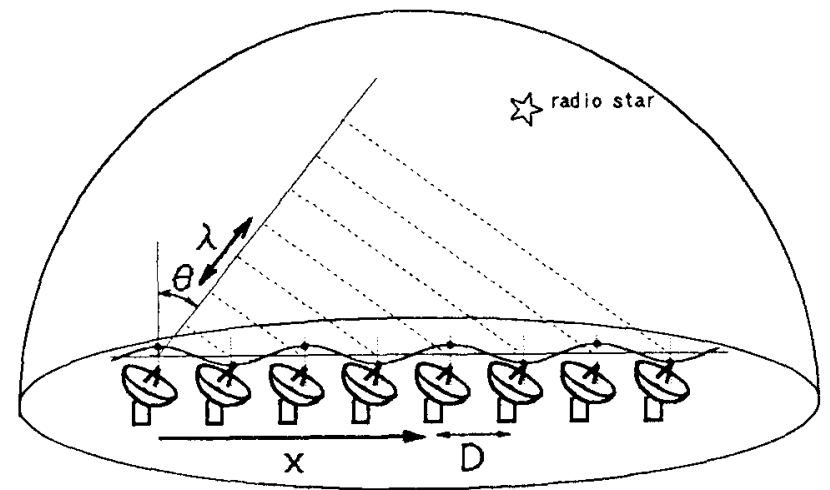

FIGURE I An east-west one-dimensional FFT interferometer.

\footnotetext{
${ }^{1}$ Transfer to Communication Research Laboratory (Apr.,1993)

${ }^{2}$ Teacher of physics, Souka High Srhool
} 

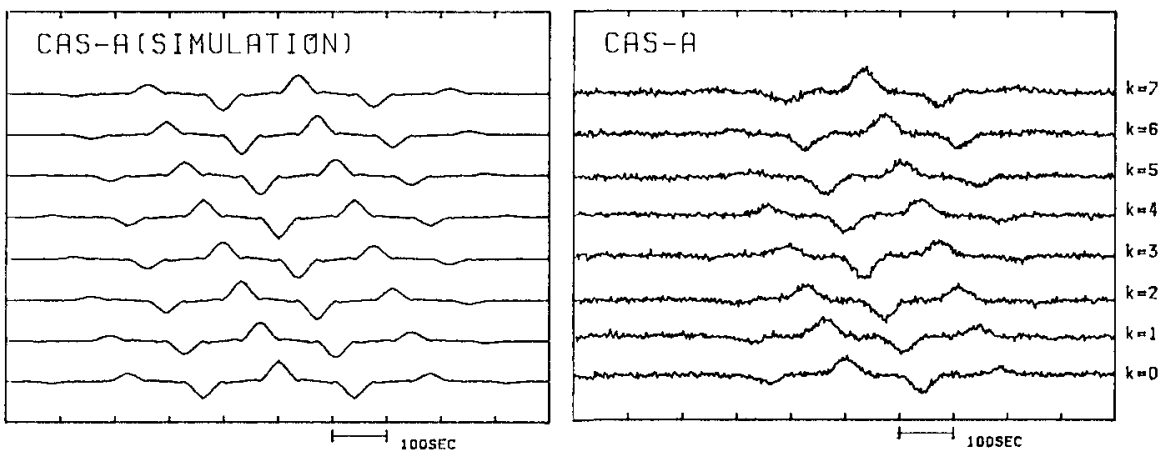

FIGURE II Simulation and result of simultaneous 8-direction observation

\section{ONE-DIMENSIONAL BEAM FORMING}

Previous to the 2D beam forming, we report a result of an east-west onedimensional (1D) expriment (Figure I). In order to measure the instrumental phase error of each element, time-domain fringes of far field sources were used (Nakajima et al. 1992). After the phase equalization, eight $1 \mathrm{D}$ beams were formed. To obtain a $2 \mathrm{D}$ image of $\mathrm{N} \times \mathrm{N}$ pixels of the sky, $\mathrm{N} \times \mathrm{N}$ equally-spaced elements sample the electric field of $E(m \Delta x, n \Delta y)$. Fourier transform of

$$
\bar{E}(k, l)=\frac{1}{N^{2}} \sum_{m=0}^{N-1} \sum_{n=0}^{N-1} E(m \Delta x, n \Delta y) e^{-i 2 \pi \frac{k}{N} m} e^{-i 2 \pi \frac{l}{N} n}
$$

gives information of radio source direction. In the presented experiment, $N-8$ in the $k$ direction and $180^{\circ}$ phase switching were used. Under the $2 \mathrm{~ms}$ phase switching, only astronomical signals are correlated. Finally the $1 \mathrm{D}$ images are obtained by

$$
P(k)=G(k)\left[\tilde{E}(k) \tilde{E}^{*}(k)-\tilde{E}_{\pi}(k) \tilde{E}_{\pi}^{*}(k)\right],
$$

where $G(k)$ is 8 -element antenna pattern and the suffix - $\pi$ indicate the applied phase gradient. We fabricated high cost-performance frontend element and observed strong radio sources in Dec., 1991. The simulation and the observed result are shown in Figure II. With the 8-elements, 8-directions differential images were obtained simulataneously. Nakajima et al. 1993 gives the details of this observation. We plan to start the $2 \mathrm{D}$ operation and the mapping in 1993.

\section{REFERENCES}

Daishido, T., Ohkawa, T., Yokoyama, T., Asuma, K., Kikuchi, H., Nagane, K., Hirabayashi, H., and Komatsu, S. 1984, in Indirect Imaging, ed. by J. A. Roberts, (Cambridge University Press, Cambridge), p. 81.

Nakajima, J., Otobe, E., Nishibori, K., Watanabe, N., Asuma. K., and Daishido, T. 1992, Publ. Astron. Soc. Japan, Vol. 44 No. 3, L35-38.

Nakajima, J. et al. $1993, p_{u b l}$. Astron. Soc. Japan, Vol. 45, in press. 